\title{
Abnormal Dispersion of Ventricular Repolarization as a Risk Factor in Patients with Human Immunodeficiency Virus: Tp-e Interval, Tp-e/QTc Ratio
}

\author{
Emine Unal Evren ${ }^{\mathrm{a}}$ Elif Ijlal Cekirdekci ${ }^{\mathrm{b}}$ Hakan Evren $^{\mathrm{a}}$ Kaya Suer $^{\mathrm{c}}$ \\ Figen Sarigul Yildirim ${ }^{d}$ Ali Asan ${ }^{e}$ Baris Bugan ${ }^{b}$
}

${ }^{a}$ Department of Clinical Microbiology and Infectious Disease, University of Kyrenia, Kyrenia, Cyprus; ${ }^{\mathrm{b}}$ Department of Cardiology, University of Kyrenia, Kyrenia, Cyprus; ${ }^{\circ}$ Department of Clinical Microbiology and Infectious Disease, Near East University, Nicosia, Cyprus; ${ }^{\mathrm{d}}$ Department of Clinical Microbiology and Infectious Disease, University of

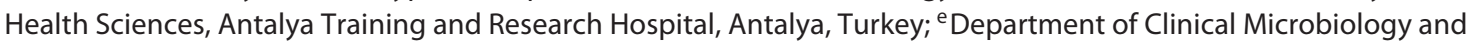
Infectious Disease, University of Health Sciences, Bursa Yuksek Ihtisas Education and Research Hospital, Bursa, Turkey

\section{Highlights of the Study}

- The cTp-e interval and the Tp-e/QT and Tp-e/corrected QTC (QTc) ratios were found to be prolonged in Human Immunodeficiency Virus (HIV)-infected patients as compared to healthy subjects.

- There was an inverse correlation between cTp-e interval, Tp-e/QTc ratio, and CD4 count, and a correlation between cTp-e interval, Tp-e/QTc ratio, and high-sensitivity C-reactive protein (hsCRP).

- Prolonged cTp-e interval, Tp-e/QTc ratio, and abnormal dispersion of ventricular repolarization may suggest an increased risk for ventricular arrhythmias and mortality in HIV-infected individuals.

\section{Keywords}

Arrhythmia · Human Immunodeficiency Virus •

Repolarization abnormalities

\begin{abstract}
Objective: In recent years, there has been worldwide recognition of the problems associated with Human Immunodeficiency Virus (HIV) infection and Acquired Immune Deficiency Syndrome (AIDS). The prevalence of cardiovascular disease in the HIV-infected population is increasing. Repolarization abnormalities, the significant contributor to life-threatening arrhythmias and mortality, are the most frequent electrocar-
\end{abstract}

karger@karger.com www.karger.com/mpp

Karger $\stackrel{\text { ' }}{5}$

GOPEN ACCESS
(C) 2020 The Author(s)

Published by S. Karger AG, Basel

This is an Open Access article licensed under the Creative Commons Attribution-NonCommercial-4.0 International License (CC BY-NC) (http://www.karger.com/Services/OpenAccessLicense), applicable to the online version of the article only. Usage and distribution for commercial purposes requires written permission. diographic changes in this population. This study aimed to evaluate the changes in Tp-e interval, Tp-e/QT and Tp-e/corrected QT (QTc) ratios, and traditional electrocardiographic features of electrical dispersion in adults infected with HIV. Subjects and Methods: A total of 235 participants were selected in the current study. The HIV group consisted of 85 subjects (median age 36 years [25-48], and the control group included 150 individuals (median age 39 years [27-51]). Tp-e interval, Tp-e/QT and Tp-e/QTc ratios were measured by the 12-lead electrocardiogram. Results: Tp-e interval, cTp-e interval, and Tp-e/QT and Tp-e/QTc ratios were significantly higher in HIV patients compared to the control group ( $p=$ $0.006, p=0.004, p=0.003$, and $p=0.002$, respectively). In 
correlation analysis, there was inverse correlation between the mean cTp-e interval and CD4 count and Tp-e/QTc ratios and CD4 count $(r=-0.407, p<0.001, r=-0.416, p<0.001$, respectively). Besides, there was correlation between the mean cTp-e interval and high-sensitivity $C$-reactive protein (hsCRP) and Tp-e/QTc ratios and hsCRP $(r=0.403, p=0.001$, $r=0.406, p=0.001$, respectively). Conclusion: Our study revealed that the cTp-e interval, Tp-e/QT and cTp-e/QT ratios were prolonged and correlated to the severity of the disease in HIV-infected patients. Our findings may shed light on the cTp-e interval and Tp-e/QTc ratio and lead to further studies showing a relationship with ventricular arrhythmias and mortality in HIV-infected individuals.

(C) 2020 The Author(s)

Published by S. Karger AG, Basel

\section{Introduction}

Human Immunodeficiency Virus (HIV) infection and Acquired Immune Deficiency Syndrome (AIDS) represent a continued serious concern to healthcare providers with a current estimated number of 40 million people living with the virus worldwide [1]. The clinical manifestations of HIV infection range from an asymptomatic state to severe immunodeficiency and serious secondary conditions [2]. It has been shown that cardiac involvement, such as coronary artery disease, myocarditis, cardiomyopathy, and arrhythmias in HIV/AIDS patients are prevalent $[3,4]$. An increased prevalence of electrocardiographic (ECG) abnormalities, including atrial fibrillation, sinus tachycardia, non-specific ST segment and T wave changes, and QT interval prolongation associated with arrhythmias and sudden death in HIV-infected subjects were previously described $[5,6]$.

Increased dispersion of repolarization, the disorder of the normal pattern of ventricular recovery, is generally known to be a marker of ventricular arrhythmias. Ventricular repolarization can be evaluated by QT, and corrected QT (QTc) interval and $\mathrm{T}$ wave analysis. The interval between the peak and the end of the T wave on ECG has been shown to be an index of total dispersion of repolarization $[7,8]$. In comparison with a single assessment of either Tp-e or QT interval, Tp-e/QT ratio is found to be a more sensitive sign of arrhythmias because the ratio stays steady while others may change according to the dynamic changes in the heart rate [9].

This study aimed to evaluate the changes in Tp-e interval, Tp-e/QT ratio, Tp-e/QTc ratio, and traditional ECG features of electrical dispersion in adults infected with HIV.

Abnormal Dispersion of Ventricular

Repolarization

\section{Materials and Methods}

This multi-center, retrospective study conducted between January 1,2016, and December 31, 2018, focused on patients infected with HIV. The following data were collected from the patient records: age, gender, clinical presentation, electrocardiographic, and echocardiographic imaging.

Power analyses of the study were performed using the program of $G^{*}$ Power (version 3.1.9.2) power-and-sample size calculation (Düsseldorf Universität, Germany). The sample size was estimated based on the possible number of individuals that could be recruited in a reasonable time with a 2:1 allocation ratio. According to the calculation, the minimum sample size was 198 individuals for all groups to detect differences between results with a statistical power (1- $\beta$ value) of $95 \%$ allowing for a type $(\alpha)$ error of 0.05 . Of those 198 individuals, 66 were required for the HIV-infected group and 132 for the control group. A total of 235 participants were selected in the current study. The HIV group consisted of 85 newly diagnosed subjects ( 58 male, age $25-48$ years [median 36 ]), and the control group included 150 individuals (101 male, age $27-51$ years [median 39]).

Patients with valvular heart disease, documented coronary artery disease, hypertension, diabetes mellitus, chronic renal/kidney failure, chronic lung disease, other congenital heart diseases, pulmonary hypertension, pregnancy, malignancy, electrolyte imbalance, paced rhythms, bundle branch block, or atrioventricular conduction abnormalities were excluded from the study. Additionally, the patients who were using any medication, including antihypertensive, antidiabetic agents, antiarrhythmic drugs, and antiretroviral drugs, that could affect the ECG measurements at the time of admission were not selected for the study. The CD4+ lymphocyte counts were gathered from the patients' medical records. Informed consent was obtained from the control group involved in this study.

Blood samples were drawn from the antecubital vein at admission after a fasting period of $12 \mathrm{~h}$ and collected in standardized tubes containing dipotassium ethylenedinitrilo tetraacetic acid for complete blood count. Glucose, creatinine, and lipid profile were determined by standard methods [10]. An automatic blood counter (Sysmex XT 2000i Hematology Analyzer; Sysmex, Kobe, Japan) was used for complete blood count.

Echocardiography was performed by using an echocardiography platform (GE Vivid 3; GE Healthcare, Piscataway, NJ, USA) equipped with a 1.5 -to-3.6- $\mathrm{MHz}$ phased-array probe in the left lateral decubitus position. The left ventricular ejection fraction was measured using the modified Simpson's rule [11].

\section{Electrocardiogram Analysis}

ECGs were recorded digitally at $10 \mathrm{~mm} / \mathrm{mV}$ calibration at a speed of $25 \mathrm{~mm} / \mathrm{s}$ for $10 \mathrm{~s}$ in the supine position. QT interval (from the onset of the QRS complex to the end of the T wave) and corrected QT interval (according to Bazett's formula [QTc $=\mathrm{QT} / \mathrm{RR}]$ ) were measured [12].

Tp-e (Tp-e tangent) interval was characterized as the time from the peak of the $\mathrm{T}$ wave to the intersection between the tangent line and the isoelectric line [13]. Tp-e (Tp-e tail) interval was characterized as the time from the peak of the $\mathrm{T}$ wave to the end of the T wave in the tail method (Fig. 1). The basic difference between the 2 methods is that the tail method has the terminal phase of the T wave. Measurements of Tp-e interval were done 
Fig. 1. Measurement of the Tp-e interval by both the tail and the tangent methods. Tp-e, interval between the peak and the end of the $\mathrm{T}$ wave.

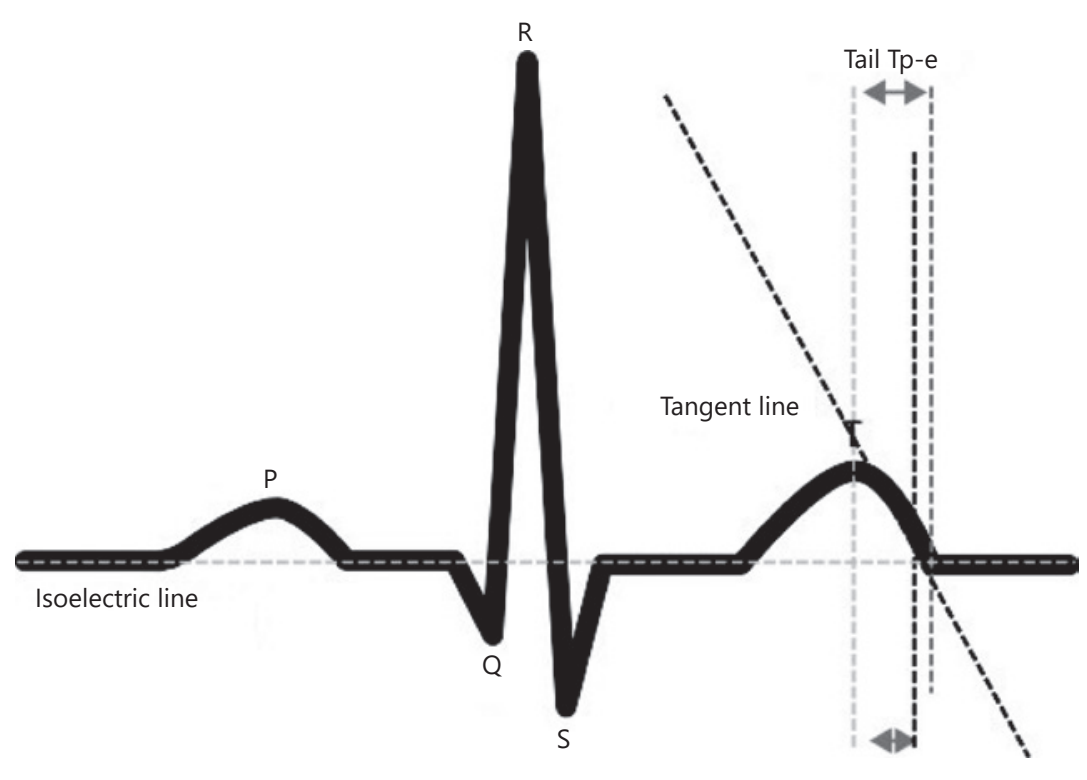

Tangent Tp-e from precordial leads (II, V2, V5), the mean value of the measurements was used in the analysis and corrected for the heart rate (cTp-e). If T-wave was low voltage, biphasic or negative, the QT interval was measured from the time of the final return of the deflection to the baseline [14]. The tail method was used for the Tp-e measurements in this article. The Tp-e/QT and Tp-e/ QTc ratios were also measured from these values. The intra- and interobserver variability of the measurements were assessed using 20 randomly selected ECGs measured by 2 independent cardiologists. Investigators were blinded as to the clinical status of the subjects. Intraobserver and interobserver coefficients of variation (SD of differences between 2 observations divided by the mean value and expressed as the percent) were 2.1 and $2.5 \%$, respectively.

\section{Statistical Analysis}

Statistical analyses were performed using SPSS software version 22 8SPSS Inc. (Chicago, IL, USA). The variables were investigated using visual (histograms, probability plots) and analytical methods (Kolmogorov-Smirnov/Shapiro-Wilk test) to determine whether or not they were normally distributed. Continuous variables were presented as mean $\pm \mathrm{SD}$ or medians (interquartile ranges), whereas categorical variables were summarized as the number of cases with the percentage (\%). Overall comparisons of categorical variables were performed using Pearson's $\chi^{2}$ test and Fisher's exact test. The Student $t$ test was used for normally distributed parameters, whereas the Mann-Whitney $U$ test was used for the parameters not distributed normally. Pearson correlation analysis was performed to examine the relationship between mean cTp-e interval, Tp-e/QTc ratio, and CD4 count and high-sensitivity Creactive protein (hsCRP). A $p$ value $<0.05$ was accepted as statistically significant.

\section{Results}

The baseline clinical data and laboratory parameters are presented in Table 1. The study population was divided into 2 groups, which were similar regarding sex distribution, age, BMI, smoking status, and baseline laboratory findings $(p>0.05)$. The ECG parameters of the groups are shown in Table 2 . The $\mathrm{QRS}, \mathrm{RR}$, and heart rate were similar between the groups $(p=0.414, p=0.787$, and $p=0.738$, respectively). QTc interval was significantly increased in HIV patients compared to the controls ( $p=$ 0.033). Tp-e interval, cTp-e interval, Tp-e/QT ratio, and Tp-e/QTc ratio were also significantly higher in HIV-infected patients compared to the control group $(p=0.006$, $p=0.004, p=0.003$, and $p=002$, respectively). There were no significant differences between the 2 groups regarding their ejection fraction $(p=0.876)$. In correlation analysis, there was an inverse correlation between mean cTp-e in- 
Table 1. Clinical characteristics and laboratory findings of the study population

\begin{tabular}{lccl}
\hline Variables & $\begin{array}{c}\text { HIV patients } \\
(n=85)\end{array}$ & $\begin{array}{l}\text { Control group } \\
(n=150)\end{array}$ & $\begin{array}{l}p \\
\text { value }\end{array}$ \\
\hline Age, years & $36(25-48)$ & $39(27-51)$ & 0.722 \\
Male & $58(68)$ & $101(63)$ & 0.117 \\
Smoking, \% & 36 & 29 & 0.268 \\
BMI & $22.5 \pm 2.3$ & $23.1 \pm 4.4$ & 0.243 \\
Systolic BP, mm Hg & $123 \pm 7$ & $125 \pm 11$ & 0.132 \\
Diastolic BP, mm Hg & $79 \pm 6$ & $77 \pm 9$ & 0.168 \\
Hemoglobin, g/dL & $13.1 \pm 2.4$ & $12.8 \pm 3.6$ & 0.493 \\
Glucose, mg/dL & $94.5 \pm 7.1$ & $95.2 \pm 8.3$ & 0.514 \\
Urea, mg/dL & $13.8 \pm 3.4$ & $13.5 \pm 2.1$ & 0.404 \\
Serum creatinine, mg/dL & $0.9 \pm 0.2$ & $0.8 \pm 0.6$ & 0.137 \\
Total cholesterol, mg/dL & $182.1 \pm 35.8$ & $174.6 \pm 43.4$ & 0.177 \\
LDL cholesterol, mg/dL & $124.5 \pm 33.8$ & $131.2 \pm 41.4$ & 0.205 \\
HDL cholesterol, mg/dL & $43.7 \pm 11.2$ & $41.8 \pm 9.4$ & 0.166 \\
Triglycerides, mg/dL & $152.4 \pm 33.8$ & $149.2 \pm 35.4$ & 0.499 \\
hsCRP, mg/dL & $5(2-7)$ & - & - \\
CD4 count, cells/mm ${ }^{3}$ & $210(82-544)$ & - & - \\
\hline
\end{tabular}

Data are given as median (IQR), mean $\pm \mathrm{SD}$, or $n(\%)$, unless otherwise indicated. BP, blood pressure; HDL, high-density lipoprotein; HIV, Human Immunodeficiency Virus; LDL, low-density lipoprotein.

terval and CD4 count and Tp-e/QTc ratios and CD4 count $(r=-0.407, p<0.001$, and $r=-0.416, p<0.001$, respectively). Besides, there was a correlation between the mean cTp-e interval and hsCRP and Tp-e/QTc ratios and hsCRP $(r=0.403, p=0.001$, and $r=0.406, p=0.001$, respectively; Fig. 2).

\section{Discussion}

The most important findings that emerged from this study are that Qtc, Tp-e interval, cTp-e interval, Tp-e/QT ratio, and Tp-e/QTc ratios were prolonged in HIV-infected patients as compared to healthy individuals. Ventricular repolarization markers, including the corrected QT interval and QT dispersion have been used for risk stratification in various clinical settings [15]. The Tp-e interval, which is an index of the transmural dispersion of ventricular repolarization, shows the different duration of the action potential in the epicardium, endocardium, and $\mathrm{M}$ cells from the heart. Previous studies have reported that prolonged QT interval and Tp-e interval are strongly associated with sudden cardiac death probably due to an increased sensitivity to arrhythmias caused by ventricular re-entry [8]. However, the Tp-e/QTc ratio is
Table 2. Electrocardiographic and echocardiographic characteristics of the study population

\begin{tabular}{lccc}
\hline Variables & $\begin{array}{l}\text { HIV patients } \\
(n=85)\end{array}$ & $\begin{array}{l}\text { Control group } \\
(n=150)\end{array}$ & $p$ value \\
\hline Heart rate, bpm & $73.3 \pm 9.4$ & $71.6 \pm 11.1$ & 0.738 \\
RR interval, ms & $845.1 \pm 125.0$ & $852.4 \pm 134.5$ & 0.787 \\
QRS interval, ms & $92.5 \pm 13.0$ & $94.0 \pm 16.7$ & 0.414 \\
QT interval, ms & $362(344-380)$ & $357(319-390)$ & 0.331 \\
QTc interval, ms & $402(386-412)$ & $386(324-426)$ & 0.033 \\
Tp-e interval, ms & $94.6 \pm 16.4$ & $85.0 \pm 16.0$ & 0.006 \\
cTp-e interval & $107.1 \pm 18.9$ & $95.4 \pm 13.1$ & 0.004 \\
Tp-e/QT ratio & $0.26 \pm 0.04$ & $0.23 \pm 0.04$ & 0.003 \\
Tp-e/QTc ratio & $0.23 \pm 0.04$ & $0.21 \pm 0.04$ & 0.002 \\
LVEF, \% & $64.1 \pm 5.4$ & $65.1 \pm 3.5$ & 0.107 \\
\hline
\end{tabular}

Data are given as mean \pm SD or median (IQR). LVEF, left ventricular ejection fraction.

more valuable when compared to either Tp-e or QT intervals due to its steady-state, regardless of the dynamic physiological changes in the heart rate and inter-individual variation of QT interval [9]. Previous reports indicated that antiretroviral agents, including protease inhibitors and non-nucleoside reverse transcriptase inhibitors, cytochrome-P450-dependent drug interactions, opioid usage, and direct HIV virus effects on cardiac ion hERG $\mathrm{K}+$ channels could affect QT intervals and cause QT prolongation. Furthermore, Nagai et al. [16] have pointed out the relationship between the insular cortex and cardiovascular system regulation. They have mentioned that the stimulation of the posterior insular cortex induced increasing degrees of atrioventricular block producing escape rhythms, premature ventricular contractions, QT prolongation, and ultimately death in asystole. More importantly, McIntosh et al. [17] showed that QTc prolongation had an important association with distinct patterns of ventromedial prefrontal cortex state functional connectivity (rsFC) with the posterior and anterior insula. Longer QTc and lower CD4 count corresponded to weaker ventromedial prefrontal cortex connectivity with the dorsal striatum in patients with HIV.

Consistent with the literature, the present study showed that the mean QTc interval in HIV-positive patients was significantly longer than that in HIV-negative subjects. Previously, Ünal et al. [4] demonstrated that HIV-infected patients receiving combination antiretroviral therapy had a longer Tp-e interval and Tp-e/QTc ratio compared to control subjects. This study revealed that Tp-e interval, cTp-e interval, Tp-e/QT ratio, and Tp-e/ 

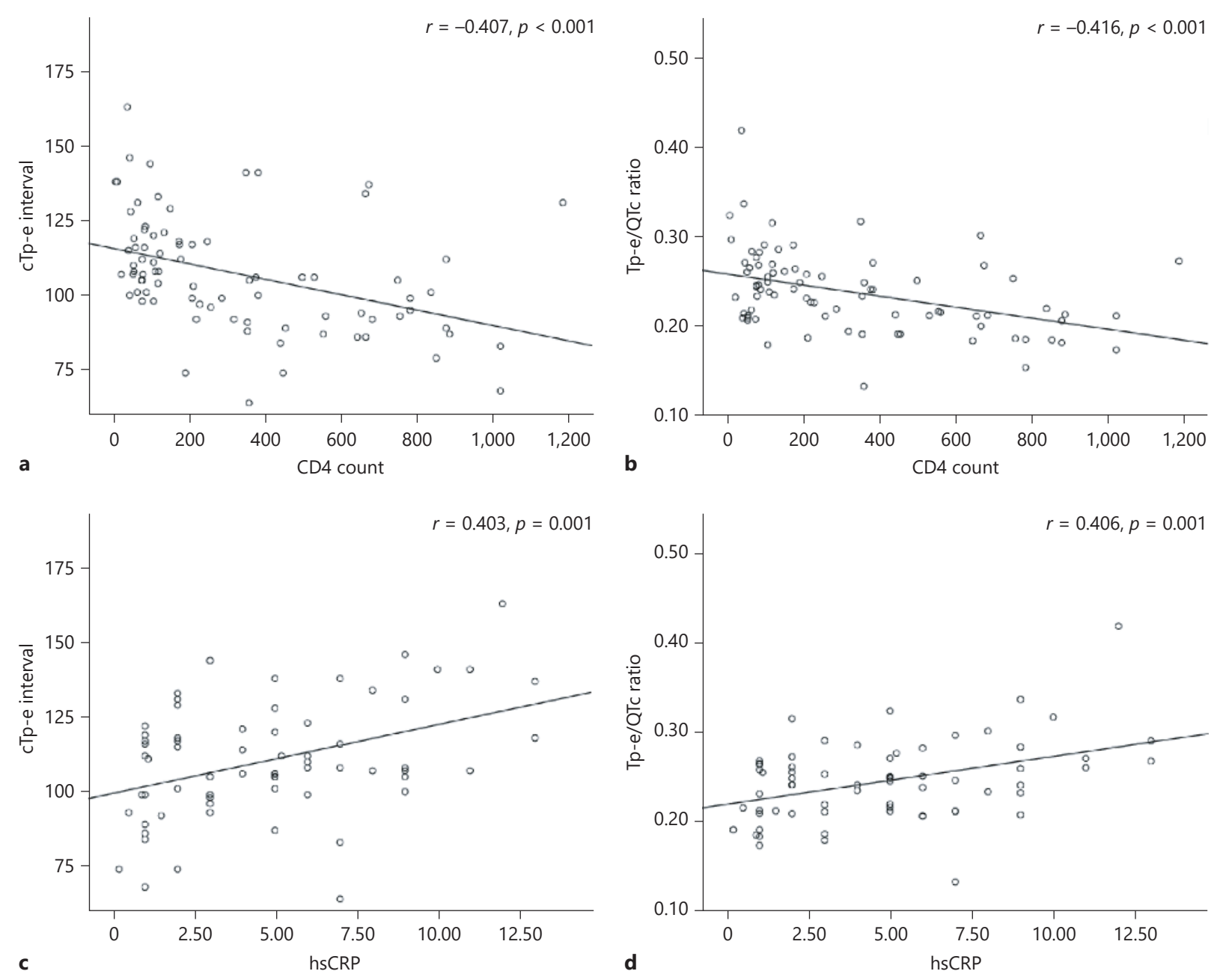

Fig. 2. a Correlation between cTp-e interval and CD4 count. b Correlation between Tp-e/QTc ratio and CD4 count. c Correlation between cTp-e interval and hsCRP. d Correlation between Tp-e/QTc ratio and hsCRP.

QTc ratio, which are the most sensitive index of ventricular repolarization and which may have led to the potentially higher risk of ventricular arrhythmia and cardiac mortality, were significantly prolonged in HIV-positive and treatment-naive patients. Hence, these results may indicate the importance of arrhythmic substrate in the hearts of drug-free HIV-infected patients. Changes in the adaptive immune system and persistent inflammation leading to severe ventricular electrophysiological remodeling could be the possible mechanisms. Indeed, experimental evidence, especially transgenic mouse models, provide a view of the pathophysiological mechanisms of HIV-related arrhythmia and strongly support the role for inflammation and pro-inflammatory cytokines (tumor necrosis factor-alpha [TNFa] and interleukin [IL]-1 $\beta$ ) on cardiac potassium and calcium ion channels and associated pathologic electrical remodeling [18-21]. IL-1 $\beta$ decreases the calcium currents, whereas TNF $\alpha$ reduces potassium and sodium currents without affecting calcium currents. Together, these events may contribute to cardiac arrhythmias in the setting of HIV infection [22].

CRP is a well-known acute-phase protein along with several cytokines such as IL-6 and TNFa [23]. In this study, the inflammatory marker hsCRP was found to have positive correlations with the cTp-e interval. Therefore, this present study is consistent with the previous results. 
Another important finding of the current study was that prolonged Tp-e intervals had a negative correlation with CD4 count. This result is in accord with previous studies, indicating that a more advanced state of the HIV infection, defined by a nadir of the CD4 cell count below 200 cells $/ \mathrm{mm}^{3}$, was the only independent predictor for ventricular repolarization prolongation in treatment-naive HIV infected patients [22]. Overall, this study could suggest that an underlying feature of HIV affects the electrical properties of the heart.

The main limitation of this study is the limited sample size and retrospective origin. The ECG features of HIVinfected patients were investigated before starting antiretroviral drug treatment. The subsequent follow-up of these patients on antiretroviral drug treatment was not applied. Additionally, due to the cross-sectional nature of the study, the patients were not followed for future arrhythmic events. Furthermore, the proinflammatory cytokines TNFa and IL-1 were not evaluated. Therefore, prospective randomized studies in a larger population are required to confirm ECG findings with incident cardiovascular events before its clinical significance can be established.

\section{Conclusion}

This study shows an abnormal dispersion of ventricular repolarization in HIV-infected patients. It also confirms previous findings and contributes additional evidence suggesting that prolonged ventricular repolarization indices may play a role in developing new risk stratification systems focusing on treatment-naïve HIVinfected individuals. Moreover, we consider that the present work is a useful starting point for future investigations of the Tp-e interval, Tp-e/QTc ratio, and HIV-related arrhythmia and mortality.

\section{Statement of Ethics}

This study was approved by the Institutional Ethics Committee. The study participants gave their informed consent for publication.

\section{Disclosure Statement}

The authors declare that they have no potential conflicts of interest concerning the research, authorship, and publication of this article.

\section{Funding Sources}

The authors received no financial support for the research, authorship, and publication of this article.

\section{Author Contributions}

All authors contributed to the conception of the work and drafted the manuscript. All authors critically revised the manuscript. All gave final approval and agreed to be accountable for all aspects of the work in ensuring that questions related to the accuracy or integrity of any part of the work are appropriately investigated and resolved.

\section{References}

1 Organization WH. Guidelines for managing advanced HIV disease and rapid initiation of antiretroviral therapy, July 2017. Geneva: World Health Organization; 2017.

2 Greenberg AE, Thomas PA, Landesman SH, Mildvan D, Seidlin M, Friedland GH, et al. The spectrum of HIV-1-related disease among outpatients in New York City. AIDS. 1992 Aug;6(8):849-59.

3 Karavidas A, Foukarakis M, Lazaros G, Chini M, Fotiadis I, Arapi S, et al. Assessment of cardiac function with Doppler tissue imaging in asymptomatic HIV-infected patients. Int J STD AIDS. 2008 Apr;19(4):22731.

4 Ünal S, Yayla Ç, Açar B, Ertem AG, Akboğa MK, Gökaslan S, et al. Tp-e interval and Tp-e/ QT ratio in patients with Human Immunodeficiency Virus. J Infect Public Health. 2018 Jan-Feb;11(1):35-8.
5 Sani MU, Okeahialam BN. QTc interval prolongation in patients with HIV and AIDS. J Natl Med Assoc. 2005 Dec;97(12): 1657-61.

6 Reinsch N, Buhr C, Krings P, Kaelsch H, Neuhaus K, Wieneke $\mathrm{H}$, et al.; German Heart Failure Network. Prevalence and risk factors of prolonged QTc interval in HIVinfected patients: results of the HIV-HEART study. HIV Clin Trials. 2009 Jul-Aug;10(4): 261-8.

7 Kors JA, Ritsema van Eck HJ, van Herpen G. The meaning of the Tp-Te interval and its diagnostic value. J Electrocardiol. 2008 NovDec;41(6):575-80.

8 Antzelevitch C, Sicouri S, Di Diego JM, Burashnikov A, Viskin S, Shimizu W, et al. Does Tpeak-Tend provide an index of transmural dispersion of repolarization? Heart Rhythm. 2007 Aug;4(8):1114-6.
9 Gupta P, Patel C, Patel H, Narayanaswamy S, Malhotra B, Green JT, et al. T(p-e)/QT ratio as an index of arrhythmogenesis. J Electrocardiol. 2008 Nov-Dec;41(6):567-74.

10 Welty TK, Lee ET, Yeh J, Cowan LD, Go O, Fabsitz RR, et al. Cardiovascular disease risk factors among American Indians. The Strong Heart Study. Am J Epidemiol. 1995 Aug; 142(3):269-87.

11 Schiller NB, Shah PM, Crawford M, DeMaria A, Devereux R, Feigenbaum H, et al. Recommendations for quantitation of the left ventricle by two-dimensional echocardiography. American Society of Echocardiography Committee on Standards, Subcommittee on Quantitation of Two-Dimensional Echocardiograms. J Am Soc Echocardiogr. 1989 Sep-Oct; 2(5):358-67. 
12 Rautaharju PM, Surawicz B, Gettes LS, Bailey JJ, Childers R, Deal BJ, et al.; American Heart Association Electrocardiography and Arrhythmias Committee, Council on Clinical Cardiology; American College of Cardiology Foundation; Heart Rhythm Society; Endorsed by the International Society for Computerized Electrocardiology. AHA/ACCF/ HRS recommendations for the standardization and interpretation of the electrocardiogram: part IV: the ST segment, T and U waves, and the QT interval: a scientific statement from the American Heart Association Electrocardiography and Arrhythmias Committee, Council on Clinical Cardiology; the American College of Cardiology Foundation; and the Heart Rhythm Society. J Am Coll Cardiol. 2009 Mar;53(11):982-91.

13 Erikssen G, Liestøl K, Gullestad L, Haugaa $\mathrm{KH}$, Bendz B, Amlie JP. The terminal part of the QT interval (T peak to $\mathrm{T}$ end): a predictor of mortality after acute myocardial infarction. Ann Noninvasive Electrocardiol. 2012 Apr; 17(2):85-94.
14 Goldenberg I, Moss AJ, Zareba W. QT interval: how to measure it and what is "normal". J Cardiovasc Electrophysiol. 2006 Mar;17(3): 333-6.

15 Barr CS, Naas A, Freeman M, Lang CC, Struthers AD. QT dispersion and sudden unexpected death in chronic heart failure. Lancet. 1994 Feb;343(8893):327-9.

16 Nagai M, Hoshide S, Kario K. The insular cortex and cardiovascular system: a new insight into the brain-heart axis. J Am Soc Hypertens. 2010 Jul-Aug;4(4):174-82.

17 McIntosh RC, Chow DC, Lum CJ, Hidalgo M, Shikuma CM, Kallianpur KJ. Reduced functional connectivity between ventromedial prefrontal cortex and insula relates to longer corrected QT interval in HIV+ and HIV- individuals. Clin Neurophysiol. 2017 Oct; 128(10):1839-50.
18 Brouillette J, Grandy SA, Jolicoeur P, Fiset C. Cardiac repolarization is prolonged in $\mathrm{CD} 4 \mathrm{C} /$ HIV transgenic mice. J Mol Cell Cardiol. 2007 Aug;43(2):159-67.

19 Grandy SA, Fiset C. Ventricular K+ currents are reduced in mice with elevated levels of serum TNFalpha. J Mol Cell Cardiol. 2009 Aug; 47(2):238-46.

20 Jiang Y, Chai L, Fasae MB, Bai Y. The role of HIV Tat protein in HIV-related cardiovascular diseases. J Transl Med. 2018 May;16(1): 121.

21 Monsuez JJ, Escaut L, Teicher E, Charniot JC, Vittecoq D. Cytokines in HIV-associated cardiomyopathy. Int J Cardiol. 2007 Aug;120(2): $150-7$.

22 Brouillette J, Cyr S, Fiset C. Mechanisms of Arrhythmia and Sudden Cardiac Death in Patients With HIV Infection. Can J Cardiol. 2019 Mar;35(3):310-9.

23 Kushner I. The phenomenon of the acute phase response. Ann N Y Acad Sci. 1982;389: $39-48$. 